\title{
IL CONCETTO D'UMILTÀ NEL LINGUAGGIO DI SANTA TERESA DI GESÙ BAMBINO
}

\begin{abstract}
A bstract. Ciesielka Joanna, $l l$ concetto d'umiltà nel linguaggio di santa Teresa di Gesù Bambino [The concept of humbleness in the language of St. Theresa of Lisieux]. Studia Romanica Posnaniensia, Adam Mickiewicz University Press, Poznań, vol. XXXI: 2004, pp. 183-196. ISBN 83-232-1353-4. ISSN 0137-2475.

This paper deals with the concept of humbleness in St. Theresa of Lisieux on the basis of her texts. Firstly, the author presents the semic analysis of the adjectives and nouns determining human qualities and attitudes. Based on the analysis, the author determines 3 semantic fields: the field of lack, the field of oblivion and the field of inferiority. Then the author aims to present the unique concept of humbleness according to St. Theresa of Lisieux: observing herself as an imperfect being, wishing to remain so, her joy of being imperfect and perceiving it as a source of good.
\end{abstract}

\section{PREMESSA}

Tra le funzioni del linguaggio, una delle più importanti o la più importante è quella che permette all'uomo di esprimersi. Già nelle concezioni di W. von Humboldt la lingua viene presentata come un legame che unisce il mondo interiore con quello esteriore. Dato che, dal punto di vista comparativo, le differenze tra le lingue determinano $i$ diversi modi in cui viene percepita ed espressa la realtà, anche nell'ambito di una lingua si osservano variazioni in rapporto all'uso individuale'.

Queste variazioni, cioè: la scelta dei termini, quella dell'attualizzazione di certi tratti dell'oggetto di cui parla il soggetto, l'uso di una tale, e non altra, concatenazione di parole, e infine il giudizio così espresso vengono determinati dal punto di vista costituito dall'insieme dei fattori culturali e personali.

In questo articolo si prenderanno in esame gli scritti di santa Teresa di Lisieux nello scopo di sottoporre ad analisi il linguaggio a cui ricorre questa giovane

${ }^{1}$ Cfr. M. Dardano, P. Trifone, 1997 , p. 700. 
carmelitana per esprimere il concetto d'umiltà. Però, vista l'ampiezza del problema in cui ci addentreremo, trascureremo l'uso delle figure stilistiche che appaiono nei suoi testi. Il corpus, che costituisce la fonte degli esempi per la nostra indagine, è stato elaborato sulla base degli scritti autobiografici di santa Teresa di Lisieux (A, B e C), delle sue poesie scritte per diverse occasioni e dalle lettere rivolte alla sua famiglia, alle religiose e ai suoi "fratelli" missionari. $\mathrm{Ci}$ siamo serviti altresì di Novissima Verba, la raccolta delle ultime parole di Teresa, pronunciate durante la sua malattia nei mesi da maggio fino a settembre 1897 , scritte dalla m. Agnese di Gesù.

\section{DEFINIZIONE DI UMILTÀ}

La parola umiltà (fr. humilité) viene dal latino humilitas il quale per la sua etimologia si riferisce a humus 'terra, suolo' e humilis 'piccolo, concavo', 'misero', 'di origine non nobile'.

Nel Vocabolario della lingua italiana di Nicola Zingarelli l'umiltà viene definita come:

Caratteristica, condizione di chi (o di ciò che) è umile: casa, cibo di grande umiltà (...), consapevolezza dei propri limiti: l'umiltà è una virtù cristiana; la vostra è falsa umiltà; cstrema deferenza, reverenza, sottomissione e sim.: presentarsi a qc. con grande umiltà, in atteggiamento di profonda umiltà; modestia e riservatezza dei modi, del contegno e sim.: ella si va, sentendosi laudare / benignamente d'umiltà vestuta (DANTE).

Da umile può essere caratterizzato ciò che è poco elevato da terra (pianta, capanna umile; umili arbusti); poco elevato, quanto a grado sociale (umile condizione; umili origini); povero, modesto, dimesso (un'umile casetta; un umile impiego). Si osserva l'uso di questo aggettivo nel significato di meschino, misero, vile: (i lavori più umili; umili servizi). Umile è colui che è pienamente consapevole dei propri limiti e non si inorgoglisce per le proprie qualità, virtù o meriti personali o per i successi che consegue, né tanto meno ricerca la fama, la gloria, la ricchezza e sim. (una persona umile; un eroe umile; essere umile di cuore); che riconosce e accetta l'autorità o la supremazia altrui e si comporta con rispettosa sottomissione (essere umile con tutti; ecco l'umile servo di Dio). Da umile può essere anche definito colui che si abbassa, si umilia per paura, per muovere gli altri a pietà e sim. (farsi tutto umile).

Gli autori del Dizionario Garzanti della lingua italiana riferiscono la nozione d'umiltà esplicitamente al cristianesimo. L'umiltà viene definita come:

Virtù cristiana che consiste nel riconoscimento della propria limitatezza e miseria di fronte a Dio; sentimento di riverenza; contegno sottomesso: non solo si deve predicare, ma anche praticare l'umiltà; pregare Dio con umiltà. 
Visto il contesto in cui è stato scritto il materiale per la nostra analisi, è necessario, a nostro avviso, puntare l'attenzione proprio sull'accezione cristiana della parola "umiltà".

Alla luce dell' insegnamento della Chiesa cattolica, l'umiltà traduce l'atteggiamento dell'uomo peccatore verso Dio. Secondo essa, l'uomo umile si rende conto che tutto quello che possiede, I'ha ottenuto da Dio. Si crede di essere un servitore inutile che non è capace di fare alcuna cosa di sé stesso. E' peccatore ma Dio innalzerà gli umili che si aprono all'azione trasformante della grazia ${ }^{2}$.

R. Grzegorczykowa, nella sua analisi dedicata alla nozione d'umiltà (1993), sottolinea la presenza di tre elementi nel nucleo semantico della comprensione cristiana di questa virtù: la coscienza della propria debolezza e la coscienza della dipendenza da Dio, l'accettazione di questo stato e, in conseguenza, la condotta rispetto agli altri risultante da essa.

L'importanza dell'umiltà nella vita cristiana risulta dall'invito di Cristo a imitarlo, espresso nelle parole: "Io sono la via, la verità e la vita; nessuno viene al Padre se non per mezzo di me" (Giovanni 14,6) e anche "Imparate da me che sono mite ed umile di cuore e troverete riposo alle anime vostre " (Mt 11, 29) Questa richiesta significa per i cristiani la necessità di abbassarsi davanti a Dio. Inoltre, l'atteggiamento umile costituisce le fondamenta per lo sviluppo dell' amore.

\section{L'ANALISI COMPONENZIALE}

La definizioni sopraccitate, dell'umiltà intesa come un atteggiamento umano consistente in un certo tipo di valutazione di se stessi e degli altri ${ }^{3}$, accennano la presenza in essa di quattro componenti importanti: l'atto del valutare, il soggetto che valuta, il soggetto o l'azione sottoposti alla valutazione, il punto di riferimento (Dio) al cui cospetto si svolge questo processo.

Dato che gli scritti di santa Teresa sono un testo religioso, il modo in cui la loro autrice intende alcuni termini che usa per descrivere se stessa, differisce dal significato correntemente inteso di essi e viene determinato proprio da questo contesto. Vogliamo dunque puntare l'attenzione su due aspetti del significato di un termine, cioè sulla denotazione e sulla connotazione. Nel supplemento al vocabolario di lingua francese, Le Robert, la denotazione viene definita come il significato di base di una parola ${ }^{4}$. Invece la connotazione è costituita da un insieme di associazioni individuali, cioè di tutto quello che un termine può evocare o

\footnotetext{
${ }^{2}$ Cfr. X. Léon-Dufour, 1985, p. 698.

${ }^{3}$ R. Grzcgorczykowa, 1993, p. 23.

${ }^{4}$ Cli. C. Baylon, P. Fabre, 1994, p. 40.
} 
suggerire in modo vago o preciso. Essa è un valore particolare attribuito ad un termine per il contesto e in relazione alla situazione ${ }^{5}$.

Per estrapolare il modo in cui santa Teresa intenda ed esprima il concetto d'umiltà, abbiamo dunque messo sotto esame i termini che ha utilizzato per descrivere se stessa e il suo comportamento. Però, alla luce di quello che è stato segnalato sopra, lo sfondo per la nostra ricerca era costituito dagli scritti di santa Teresa e non dal sistema linguistico. In conseguenza, alla lista dei tratti di significato da noi individuati nei termini sottoposti ad esame, abbiamo aggiunto anche alcuni virtuemi ${ }^{6}$. Nelle tabelle presentate sotto, mettiamo in rassegna alcuni tratti semici dei termini usati dalla santa, attualizzati nei suoi scritti.

\begin{tabular}{|c|c|c|c|c|c|c|c|c|c|c|c|c|c|c|c|c|c|c|c|c|c|c|c|c|c|}
\hline & 氮 & 章 & 氧 & ㄴ. & 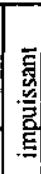 & 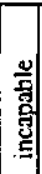 & 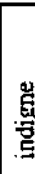 & 츌 & 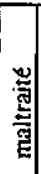 & 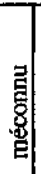 & 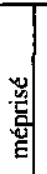 & 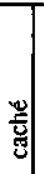 & 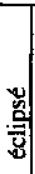 & $\begin{array}{l}\text { 잉 } \\
\text { 영 }\end{array}$ & 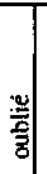 & 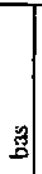 & 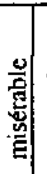 & & & & & & & & $\frac{\pi}{>}$ \\
\hline+ umano & + & & + & & + & + & + & + & + & + & + & + & + & + & + & & + & + & & + & \pm & + & & + & \\
\hline+ oggetto & & + & & + & & & & & & & - & + & & 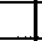 & & 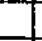 & & 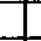 & - & 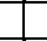 & 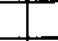 & 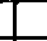 & + & & + \\
\hline+ astratto & & + & & & & & & & & & 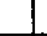 & . & & + & & + & & & + & & + & & + & & \\
\hline posizione inferiore & + & & + & + & + & + & + & & + & & + & + & + & + & + & + & + & + & + & + & + & + & & & \\
\hline che ha poco valore & + & + & + & + & & & + & + & & & + & & & & + & + & + & + & ++ & $+1+$ & ++ & + & + & & \\
\hline Mancanza di & + & + & + & + & + & + & + & + & & & & & & & & & + & + & ++ & ++ & ++ & + & + & & + \\
\hline - forza morale & + & & + & & + & + & & + & & & & & & & & & + & + & + & + & 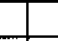 & \pm & & & \\
\hline - resistenza & + & & + & & + & + & & + & & & & & & & & & + & + & + & + & + & + & & & \\
\hline - perfezione & + & + & + & + & + & + & + & + & & & & & & & & & + & + & ++ & ++ & + & \pm & + & & \\
\hline invisibile & + & & & & & & & & & & & \pm & + & + & + & & & & & & & \pm & & & \\
\hline $\begin{array}{l}\text { che non è preso in } \\
\text { considemzione }\end{array}$ & + & & & & & & & & + & & + & +1 & + & + & + & +1 & + & & & & & + & & & \\
\hline male & + & & & & & & & + & + & + & + & & & & & & & & & & & & & & \\
\hline sconosciuto & + & & & & & & & & - & + & - & & + & + & + & & . & & & & & 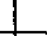 & & & בـ \\
\hline modesto & + & & & + & + & & & & + & + & & + & + & & & + &.+ & + & $+1+$ & + & & + & + & + & + \\
\hline senza merilf & + & & + & & & & + & + & & + & + & & & + & + & + & + & + & + & + & + & + & & & + \\
\hline che ispira la pietà & & & + & & + & + & & & \pm & & & & & & & & +1. & \pm & \pm & + & & & & & 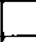 \\
\hline $\begin{array}{l}\text { che non possiede } \\
\text { nulla }\end{array}$ & & & & & & & & & & & & & & & & & & + & & & & + & & & + \\
\hline $\begin{array}{l}\text { che ha bisogno } \\
\text { dell'aiuto di Dio }\end{array}$ & + & & + & & + & + & + & + & & & & + & + & & + & + & +1 & + & & + & + & + & & + & + \\
\hline
\end{tabular}

Dalla nostra analisi risulta che non esiste un semema comune a tutti i sostantivi e a tutti gli aggettivi presentati sopra affinché sia possibile farne un solo campo semantico. Ne possono essere individuati molti, però, sotto ne presentiamo tre, che sono costituiti attorno ai tratti che appaiono nella maggior parte dei vocaboli. Questj tratti sono: la mancanza, la scomparsa e la bassezza. Da segnalare rimane ancora che a volte questi tratti si manifestano insieme.

${ }^{5}$ Ibidem, p. 40.

${ }^{6}$ lbidem, p. 53. 


\begin{tabular}{|c|c|c|c|c|c|c|c|c|c|c|c|c|c|c|c|c|c|c|c|c|c|c|}
\hline & 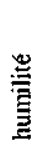 & 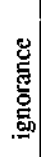 & 产 & 莺 & 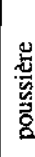 & 롤 & 言 & 䓌 & 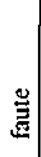 & 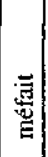 & 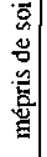 & 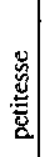 & 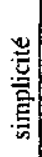 & 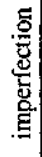 & 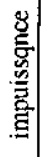 & 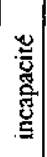 & 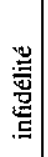 & 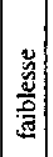 & 营 & 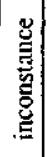 & 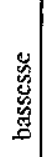 & 岂 \\
\hline persona & & & & + & & & & & & & & & & & & & & & & & & \\
\hline $\cos 3$ & & & & & + & + & & & & & & & & & & & & & & & & \\
\hline stato & & + & & & & & & & & & & & & & & + & & + & & & + & + \\
\hline attitudine & + & & & & & & & & & & + & & & & & & & & + & & & \\
\hline tratto & + & & & & & & & + & & & & + & + & + & + & + & + & + & & + & & \\
\hline azione & & & & & & & & & & + & & & & & & & + & & & & & \\
\hline risultsto dell' azione & & & + & & & & + & & + & + & & & & & & 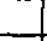 & & & & & & \\
\hline inferiorita & + & & & + & + & + & + & ( & 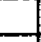 & . & + & + & & + & + & + & & + & + & & + & + \\
\hline che ha poco valore & & & & + & + & + & & & & & & + & & + & & 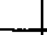 & & + & + & & + & + \\
\hline mancanza di & + & & & & + & + & + & + & + & + & & + & & + & + & + & + & + & + & + & + & + \\
\hline - forza morale & + & & & & + & + & + & + & + & + & & + & & + & + & - & + & + & & + & + & + \\
\hline - resistenza & & & & & + & + & & \pm & + & & & \pm & & + & + & 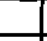 & + & + & 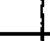 & + & + & + \\
\hline - perfezione & & & & & + & + & + & + & + & + & & + & & + & + & + & + & + & + & \pm & + & + \\
\hline invisibilita & + & + & + & & + & + & & & & & & + & & & & - & & & & & - & \\
\hline $\begin{array}{l}\text { che non è preso in } \\
\text { considerazione }\end{array}$ & + & & + & + & + & + & & & & & + & + & & & & + & & & & & + & + \\
\hline male & + & & & & & & + & + & + & + & + & + & & & & & + & + & & & & + \\
\hline sconosciuto & + & + & + & & + & + & & & & & & 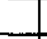 & & & & & & 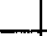 & & & & 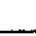 \\
\hline modesto & + & & & + & + & + & & & & & & + & + & & + & & & + & & & + & + \\
\hline senza meriti & + & & + & + & + & + & & & & & & + & & + & 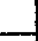 & 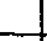 & & + & + & & + & + \\
\hline che ispira la pieta & + & & & & & & & & & & + & 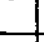 & & + & + & + & & + & & & + & + \\
\hline che non possiede nulla & & & & + & & & & & & & & + & & & 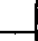 & _ & & 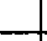 & & & + & + \\
\hline $\begin{array}{l}\text { che hil bisogno } \\
\text { dell' ajuto di Dio }\end{array}$ & + & & & + & + & + & & & & & & + & + & + & + & + & + & + & + & + & + & + \\
\hline
\end{tabular}

\section{IL CAMPO SEMANTICO DI MANCANZA/ASSENZA}

Alla luce delle definizioni presentate sopra (p. 2), una persona umile non si inorgoglisce delle proprie doti e ha coscienza dei propri limiti. Per "limiti" si intende tutto quello che allontana dall'ideale e che impedisce il suo raggiungimento. In questa prospettiva si iscrivono i vocaboli che veicolano il concetto di mancanza (diviso nella nostra tabella in: mançanza di forza morale, di resistenza e di perfezione). Questa idea viene espressa negli Scritti con dei sostantivi e aggettivi formati dall'aggiunta dei prefissi "negativi" "in-"e "im-".

Vediamo qualche esempio: imparfait 'imperfetto', imperfection 'imperfezione', impuissant 'impotente', impuissance 'impotenza', incapable 'incapace', incapacité 'incapacità', indigne 'indegno', indignité 'indegnità', inconstance 'incostanza', infidélité 'infedeltà'.

Queste forme sostantivali premesse da prefissi negativi significano la mancanza rispettivamente di: perfezione, potenza, capacità, costanza, fedeltà. 
L'autrice dichiara dunque di non possedere tutti questi tratti di solito associati alla perfezione, alla grandezza dal punto di vista della morale e della santità.

Anche gli altri termini fanno parte di questo campo. Occorre citare le parole come: faiblesse, bassesse, petitesse, misère, poussière, néant, petit, fragile, défaite, défaut, faute e méfait.

L'autrice degli Scritti vede da una parte la sua dipendenza da Dio e confessa la sua incapacità di ottenere qualsiasi cosa da se stessa; dall'altra si sente indegna delle grazie che ha ottenute.

\section{IL CONCETTO DI SCOMPARSA}

Un altro tratto particolare della comprensione teresiana dell'umiltà è la presenza nei suoi Scritti di vocaboli appartenenti al campo semantico della scomparsa: di essere allontanato dalla vista, dalla memoria e anche di non essere preso in considerazione. Tra queste parole troviamo: caché 'nascosto', éclipsé 'eclissato', ignorance 'ignoranza', ignoré 'ignorato', oubli 'oblio', oublié 'dimenticato', poussière 'polvere', néant 'nulla', mépris de soi 'disprezzo di se stesso'.

Il concetto di scomparsa si evidenzia nelle definizioni dei vocaboli sopraccitati: sia il fatto di non essere visto ("évite de se montrer ». "dérober à la vue", "rendre invisible", "ne plus être vu ou visible") sia quello di essere dimenticato, sconosciuto ("ne pas retrouver le souvenir". " $m$ c pais connaittre". "ne pas prendre en considération") . Al fatto di essere nutata, ammirata e apprezzata, Teresa preferisce la scomparsa, il nascondersi. dimenticare se stessa e essere dimenticata.

\section{IL CONCETTO DI BASSF.7.A}

Il seguente insieme di parole fa parte del campo semantico della bassezza, che acquisisce nella tabella due valori particolari: 'la posizione inferiore' e 'che ha poco valore'.

In questo campo abbiamo individuato i vocaboli che derivano dalla parola "basso" e quelli provenienti da "humilem" propr. 'basso', il quale ha per origine humus 'terra, suolo'. Negli Scritti se ne evidenzia tutta una famiglia: gli aggettivi "humble" 'umile' e "humilié" 'umiliato', i sostantivi: "humble" 'umile', "humilité" 'umiltà' e "humiliation" 'umiliazione'.

Tra i vocaboli che si evidenziano attorno ai semi 'inferiorità' e 'che ha poco valore' abbiamo trovato alcuni che indicano persone appartenenti alla cosiddetta "bassezza sociale", cioè misérable 'miserabile', pauvre 'povero', serviteur 'servo'.

Nel terzo insieme, minore rispetto gli altri, si trovano parole che indicano una cosa. Si osservi che in questo caso si adopera lo scendere fino alla base della 
piramide degli enti ${ }^{7}$ alla cui cima troviamo il concetto di Dio. Inoltre si noti che questa cosa non è qualsiasi cosa, ma proprio quella bassa ed insignificante, come lo illustrano tre esempi: "poussière" 'polvere', "néant", "rien" "nulla'. Il concetto della bassezza trova in esso il suo punto culminante perché quello che era insignificante lo diventa fino al punto di non esistere più, si annichilisce. Il campo nozionale di bassezza è il più ricco, contiene dunque anche altre parole. Include una parte dei vocaboli appartenenti al campo semantico della scomparsa e in gran parte, anche quelli formati con l'aggiunta del prefisso negativo "in".

Analizzando il problema del concetto di bassezza, si può evocare l'immagine della scala o quella dello schema della struttura sociale, comunque di elementi disposti sull'asse verticale. Questi, se trasposti sul piano orizzontale, acquisiscono il valore degli elementi contenuti tra il "primo" e l' "ultimo". Nel linguaggio della santa di Lisieux due parole di questo campo, non presentate però prima nelle tabelle, fanno parte di quest'insieme: après 'dopo', dernier 'ultimo'.

Siccome la parola "basso" evoca subito "alto", anche it vocabolo "après" evoca "avant" e "dernier" evoca "premier". La santa di Lisieux si mette dunque in opposizione a tutto ciò che si trova su posti importanti. Molto spesso quest'opposizione è implicita, ma nel caso di "après" ne abbiamo trovata una esplicita. Vediamo dunque qualche esempio:

(1) (...) en effet le zéro par lui - même n'a pas de valéur, mais placé près de l'unité il devient ptissunt, pourvu toutefois qu'il se mente de bon côté, APRÈS et non pas AVANT 1... ( LT 226, p. 410)

Anche parlando dell'ultimo posto, Teresa lo contrappone al primo:

(2) Ce n'est pas à la PREMIÈRE place, mais à la DERNİ̀RE que je m'élance; (...) (Ms C, p. 301)

\section{L'UMILTÀ DI SANTA TERESA DI GESÙ BAMBINO: VH:DERSI PICCOLA}

La presentazione del concetto d'umiltà che si riflette nel linguaggio degli Scritti di santa Teresa non sarebbe completa se mincasse il contesto più preciso in cui sono stati inseriti i vocaboli analizzati prima. Questi elementi. nella formazione di sintagmi verbali e nominali, dipendono da taluni elementi o reggono altri. In seguito al contatto con altre parole, il loro significato viene modificato o prende diverse sfumature.

Tra le proposizioni che veicolano il concetto d'umiltà, una parte viene costituita da quelle dichiarative, il cui predicato è espresso dal verbo al presente dell' indicativo, seguito da un complemento. I predicati con i verbi "essere" o "avere"

\footnotetext{
${ }^{7}$ Cfr. T.P. Krzeszowski, 1999, p. 31.
} 
seguiti dai complementi costituiscono la "descrizione" di sé stessa fatta da santa Teresa. Eccone alcuni esempi:

(3) Je devrais donc être déjà avancée dans les voies de la perfection, mais je suis encore au BAS de l'échelle; (...) (LT 202. p. 373)

(4) (...) elle est FAIBLE et bien faible, tous les jours elle en fait une nouvelle expérience (...) (LT 109, p. 179)

(5) (...) je vois que dans les occasions je suis bien IMPARFAITE. (Ms C, p. 265)

(6) En dehors de l'Office Divin que je suis bien INDIGNE de réciter, (...) (Ms C, p. 280)

(7) Depuis l'épi,j'ai des sentiments encore plus BAS de moi-même. (DE, p. 136)

(8) (...) voyant que j'ai les mêmes FAIBLESSES qu'elles, mes petites soeurs m'avouent à leur tour les fautes (...) (Ms C, p. 277)

Santa Teresa descrive se stessa e $\mathrm{i}$ suoi difetti anche in un modo molto soggettivo rispetto agli esempi sopraccitati, usando verbi, ai quali oltre alla descrizione vera e propria viene aggiunto un elemento di valutazione.

Osserviamo gli esempi:

(9) (...) elle se sentait si faible, si FRAGILE que pour jamais elle voulait s'unir à la Force Divine !... (Ms A, p. 93)

(10) (...) mais je sens mon IMPUISSANCE et je vous demande, ô mon Dieu! d'être vous même ma Sainteté. (Manuscrits autobiographiques, p. 307)

(I 1) (...) je me sens si MISÉRABLE! (DE. p. 136)

(12) (...) car là seulement se trouve la paix et le repos du caur, quand on se voit si MISÉRABLE on ne veut plus se considérer et on regarde que l'unique Bien - Aimé!... (LT 109, p. 179)

(13) (...) mais je connais mon INCAPACITÉ et je sais qu'en faisant de mon mieux je n'arriverais pas à bien faire (...) (Ms C, p. 253)

(14) Je suis la plus petite des créatures, je connais ma misère et ma FAIBLESSE, (...) (Ms B, p. 228)

L'uso degli aggettivi possessivi davanti ai sostantivi o aggettivi che introducono la nozione d'umiltà è un altro elemento che completa la descrizione dell'umiltà contenuta negli scritti di santa Teresa. La giovane carmelitana parla della sua debolezza, dei suoi errori o della sua infedeltà rispetto a Dio utilizzando l'aggettivo possessivo "mon" "mio', ma anche, nelle costruzioni metaforiche "son" 'suo'. Vediamo qualche esempio:

(15) Avec certaines âmes, je sens qu'il faut me faire petite, ne point craindre de $m$ 'humilier en avouant mes combats, mes DÉFAITES; (...) (Ms C, p. 277)

(16) Voilà pourquoi le Bon Dieu a jeté un voile sur tous mes DÉFAUTS intérieurs et extérieurs. (Ms C. p. 283) 
(17) (...) malgré mon INDIGNITÉ Il a bien voulu me prendre pour sa petite épouse. (LT 121, p. 198)

(18) Cependant après tous ses méfaits, au lieu d'aller se cacher dans un coin pour pleurer sa misère et mourir de repentir, le petit oiseau se toume vers son Bien - Aimé Soleil, (...) il raconte en détail ses INFIDÉLITÉS, (...) (Ms B, p. 231)

(19) Cependant après tous ses MÉFAITS, an lieu d'aller se cacher dans un coin pour pleurer sa misère et mourir de repentir, le petit oiseau se tourne vers son Bien-Aimé Soleil, (...) (Ms B, p. 231)

(20) (...) je suis assurée que mon Céleste Epoux suppléera à mes FAIBLES mérites (sur lesquels je ne m'appuie aucunement) (...) (LT 189, p. 340)

(21) C'èst en t'aimant que j'attire le Père/Mon FAIBLE coeur le garde sans retour. (Poésies, p. 96)

(22) (...) je suis si imparfaite que mes PAUVRES prières n'ont pas sans doute beaucoup de prix, (...) (LT 99, p. 162)

(23) (...) et votre petite fille espère aussi être dans votre couronne une toute petite fleur qui prêtera son HUMBLE éclat à la gloire de son ange visible de la terre. (LT 113, p. 184)

(24) C'est Lui seul qui se contentant de mes FAIBLES efforts, m'élèvera jusqu'à Lui (...) (Ms A, p. 86)

(25) Oubliant ma grande MISÈRE/Tu viens habiter en mon coeur. (Poésies, p. 157)

(26) C'est le bon Dieu tout sew qu'il faut faire valoir, car il n'y a rien à faire valoir dans mon petï NÉANT. (DE, p. 131)

(27) De votre Fils le regard ineffable/Sur ma PAUVRE âme a daigné s'abaisser (Poésies, p. 78)

Negli scritti della santa di Lisieux si evidenziano molti sintagmi nominali, che l'autrice riferisce a se stessa, e nei quali uno o alcuni elementi introducono la nozione d'umiltà.

Teresa si chiama: l'indigne carmélite, l'indigne sœur, pauvre Thérèse, la pauvre péchéresse, la petite solitaire, la petite fiancée ( 2 volte), la petite fille ( 6 volte), la petite créature, la petite sœur ( 7 volte), la petite éphémère, la petite carmélite ( 2 volte) une petite âme $(8$ volte), le petit zéro, petite Thérèse $(5$ volte), la plus petite de toutes les âmes, une petite sainte, une pauvre carmélite bien imparfaite, la sœur toute petite et très imparfaite, faible et imparfaite créature (2 volte), l'indigne petite sœur ( 7 volte), pauvre petit néant, âme faible et imparfaite, faible et imparfaite créature, pauvre petite novice, pauvre petite fiancée de Jésus, pauvre petite épouse, pauvre petite Thérèse (4 volte), pauvre petite sœur (2 volte), pauvre petite âme ( 2 volte), toute petite âme et très imparfaite.

A primo sguardo ci colpisce la frequenza dell'uso dell'aggettivo «petit» nelle espressioni sopraccitate. Da un lato, questa parola viene usata da Teresa per umiltà, 
dall'altra fa parte del suo modo di esprimersi, il "linguaggio teresiano", un po' infantile e sdolcinato.

Grazie all'uso degli aggettivi qualificativi, il significato dei sostantivi acquisisce una sfumatura diversa. Però, notiamo anche due casi particolari dell'uso di questi aggettivi: le petit zéro e le petit néant, in cui anche una cosa che non esiste viene qualificata da piccola.

L'uso dell'espressione "une petite sainte" che Teresa di Lisieux riferisce a se stessa può sorprendere. Ma anche questo costituisce un elemento del concetto d'umiltà nel linguaggio teresiano. Come tutti $\mathrm{i}$ cristiani, anche Teresa si sentiva chiamata a diventare santa. Però, lei si mette in una posizione inferiore rispetto ai "grandi" santi che percorrevano a volte vie difficili per ottenerla.

\section{UNO DEI DESIDERI DI TERESA: RESTARE DEBOLE}

E' da notare che, come lo precisa la nozione d'umiltà, l'atteggiamento umile non può essere limitato alla valutazione di sé fatta dal soggetto, il cui riflesso, sul piano linguistico, lo troviamo nella descrizione dei suoi tratti, azioni, ecc. L'atteggiamento umile, com'è stato detto prima, necessita anche l'accettazione di una tale condizione.

Negli scritti di santa Teresa un elemento importante lo costituiscono le frasi con un elemento volitivo. Questo gruppo comprende le proposizioni con il verbo che esprime desiderio. Questi verbi sono: "vouloir" 'volere' e "désirer" 'desiderare'.

(28) (...) en tout elle veut bien être la DERNIERE et la plus petite (...) (LT 125, p. 204)

(29) (...) je voudrais être tellement réduite au NÉANT que je n'aie aucun désir... (LT 103, p. 168)

(30) Mon Bien - Aimć. ton exemple m'imitu'A m'abaisser, à mépriser l'honneur./Pour te ravir, je veux rester petite Lin m'oubliant, je charmerai ton Coeur. (Poésies, p. 168)

(31) Oh! comme il désire d'être réduit à rien, d'être INCONNU de toutes les créatures, (...) (LT 103. p. 168)

(32) Moi aussi, je désirais être sans beauté, senle à fouler le vin dans le pressoir. INCONNUE de toute créature... (DE, p. 125)

(33) Oui je désire d'être OUBI.IÉE, el non seulement des créatures mais aussi de moimême, (...) (Lettres, LT 103. p. 168)

Come vediamo negli esempi 28-33. Teresa non solo accetta la sua condizione, ma anche non vuole che essa cambi. Nella natura umana c cè la volontà di diventare sempre più grandi, forti, potenti, di lottare contro quello che disturba. L'ambizione si trova alla base di questo desiderio. Si vuole essere belli, saggi, ammirati da tutti. Teresa invece vuole rimanere nascosta, sconosciuta, occupare sempre l'ultimo 
posto. Questo desiderio viene confermato nelle Lettere della santa in cui si osservano le frasi con modalità intenzionale imperativa. L'autrice degli Scritti si rivolge alle sue consorelle chiedendo loro che preghino per lei affinché resti dimenticata e sconosciuta. Vediamo alcuni esempi:

(34) (...) priez pour le pauvre petit grain de Sable, que le grain de sable sout toujours à sa place c'est - ̀̀ - dire sous les pieds de tous, que personne ne pense à lui, que son existence sout pour ainsi dire IGNORÉE, (...) (LT 95, p. 156)

(35) (...) demande à Jésus qu'clle soit bien fidele, qu'elle soit comme toi heureuse d'être partout la plus petite... la DERNIÈRE!... (LT 173, p. 306)

(36) Demandez que votre petite fille reste toujours un petit grain de sable bien obscur, bien caché à tous les yeux, que Jésus seul puisse le voir; qu'il devienne de plus en plus petit, qu'il soit réduit à RIEN... (LT 49, p. 86)

(37) (...) demande - Lui que je reste toujours PETTTE, toute petite!... (LT 154, p. 268)

Il desiderio di restare sempre piccola, anzi, di diventarla ancora di più, viene espresso da santa Teresa nelle sue preghiere, quando si rivolge direttamente a Dio:

(38) Que je ne cherche et ne trouve jamais que toi seul, que les créatures ne soient rien pour moi et que je ne sois RIEN pour elles mais toi Jésus sois tout!... (Manuscrits autobiographiques, p. 305)

(39) Fais que je ne sois jamais à la charge à la communauté mais que personne ne s'occupe de moi, que je sois regardée foulée aux pieds OUBLIEE comme un petit grain de sable à toi, Jésus. (Manuscrits autobiographiques, p. 305)

\section{DEBOLEZZA: GIOIA DI TERESA}

L'altro tratto che si riflette nel linguaggio della santa Teresa di Lisieux è il fatto di cercar di conciliare concetti che a primo sguardo si escludono a vicenda. La santa associa le idee della gioia e della felicità con quella delle sue imperfezioni. L'autrice degli Scritti non desiderava sopportarle come un carico. Non soltanto non voleva che causassero la sua tristezza, ma pregava per ottenere la grazia di trovarcj la gioia. Voleva essere piccola fino alla fine o piuttosto cercava la perfezione nella piccolezza. Nel suo linguaggio lo esprimono le parole della famiglia di "bonheur" 'felicità'. Ecco alcuni esempi:

(40) Quel bonheur d'être si bien cachée que personne ne pense à vous!... d'être inconnue même aux personnes qui vivent avec vous... (LT 106, p. 172)

(41) (...) j'ai reconnu par expérience que le bonheur ne consiste qu'à se cacher, à rester dans l'ignorance des choses créées. (Ms A, p. 201)

(42) (...) que ton petit oiseau est heureux d'être FAIBLE et petit, que deviendrait - il s'il était grand?... (Ms B, p. 232) 
(43) Je suis bien plus heureuse d'avoir été IMPARFAITE que si, soutenue par la grâce. j'avais été un modèle de douceur... (LT 230, p. 415)

(44) (...) je sais toujours trouver le moyen d'être heureuse et de profüter de mes MISËRES... (MS A, p. 198)

(45) Oh! que je suis heureuse de me voir IMPARFAITE et d'avoir tant besoin de la miséricorde du bon Dieu au moment de la mort! (DE, p. 102)

Negli altri frammenti si serve dei vocaboli della famiglia di «joie» 'gioia':

(46) (...) aussi maintenant je me résigne à me voir toujours IMPARFAITE et j'y trouve ma joie... (Manuscrits autobiographiques, Ms A, p. 185)

(47) J'éprouve une joie très vive non seulement lorsqu'on me trouve IMPARFAITE, mais surtout de m'y sentir moi-même. (DE, p. 119)

(48) Alors je me suis réjouie d'être PAUVRE, j'ai désiré le devenir chaque jour davantage afin que chaque jour Jésus prenne plus de plaisir à se jouer de moi, (LT 176, p. 310)

(49) Il m'arrive bien aussi des FAIBLESSES, mais je m'en réjouis. (DE, p. 59)

\section{DEBOLEZZA: SORGENTE DEL BENE}

La comprensione della debolezza come sorgente del bene è il seguente tentativo intrapreso da santa Teresa di conciliare gli elementi che sembrano inconciliabili. Le parole: "debolezza", "imperfezione", "errore" provocano piuttosto connotazioni negative. Il fatto di essere debole, piccolo, imperfetto o quello di commettere sempre errori non è un motivo di vanto. Santa Teresa non parla del senso d'impotenza o d'insoddisfazione riferendosi al suo comportamento e alla sua debolezza. Nei suoi scritti, paradossalmente, mette l'una accanto all'altra, le parole che non avremmo messo mai insieme. La debolezza non provoca in lei la scomparsa della speranza. Tutto il contrario. In un modo strano, Teresa ne trae profitti. Vediamo alcuni esempi che illustrano la nostra tesi:

(50) C'est sa FAIBLESSE qui fait toute sa CONFIANCE, il ne saurait se briser puisque, quelque chose qui lui arrive, il ne veut voir que la douce main de son Jésus... (LT 55, p. 92)

(51) Si je suis MÉPRISÉE du monde,/S'il me regarde comme un rien,/Une PAIX divine m'inonde/Car j'ai l'hostie pour mon soutien,/ (Poésies, p. 116)

(52) (...) que l'unique BIEN, c'est d'aimer Dieu de tout son coeur et d'être ici - bas PAUVRE d'esprit... (Ms A, p. 86)

(53) Mais cette PAUVRETÉ a été pour moi une vraie LUMIÈRE, une vraie GRACE. (DE, p. 126)

(54) (...) je puis donc malgré ma PETITESSE aspirer à la SAINTÉTÉ; me grandir c'est impossible, je dois me supporter telle que je suis avec toutes mes imperfections: (...) (Ms C, p. 240) 
(55) O Mère bien - aimée, malgré ma PETITESSE/Comme toi je POSSÈDE en moi Le TOUT-PUISSANT/Mais je ne tremble pas en voyant ma faiblesse:/ Le trésor de la mère appartient à l'enfant (Poésies, p. 243)

(56) Moi je me considère comme un faible petit oiseau couvert seulement d'un léger duvet; je ne suis pas un aigle, j'en ai simplement les yeux et le caur car malgré ma PETITESSE extrême j'OSE fixer le Soleil Divin. (...) (Ms B, p. 230)

(57) Je ne suis qu'une enfant, impuissante et faible, cependant c'est ma FAIBLESSE même qui me donne l'AUDACE de m'offrir en Victime à ton Amour, ô Jésus! (Ms B, p. 227)

(58) Céline, quel PRIVILÈGE d'être MÉCONNUE sur la terre!... (LT 107, p. 173)

La debolezza permette a Teresa di credere che Dio le darà tutto perché da sola non è capace di ottenere nulla. Le parole a connotazione negativa, situate nel contesto cristiano o, piuttosto, teresiano, assumono una diversa sfumatura di significato. L'autrice degli Scritti trattando le sue imperfezioni e le umiliazioni che vive come una sorgente del bene: della pace divina, della fiducia, del coraggio e della luce, attribuisce ad esse il significato positivo.

\section{CONCLUSIONE}

Come abbiamo osservato in diversi esempi sottoposti all'analisi in questo articolo, il concetto d'umiltà nel linguaggio di santa Teresa di Gesù Bambino appare attraverso gli elementi riuniti attorno ai seguenti gruppi:

1) la descrizione di se stessa e delle sue azioni fatta dalla santa,

2) l'accettazione di questo stato,

3) il desiderio che la situazione non cambi,

4) la gioia causata dalle imperfezioni e dalla debolezza,

5) l'intendimento dell' imperfezione come una sorgente del bene.

Dalle nostre analisi risulta che santa Teresa di Lisieux giudica severamente se stessa, il suo comportamento e le sue azioni. A volte designa quello che fa con le parole ad una forte connotazione negativa (mépriser, méfait). Il suo intendimento del concetto d'umiltà non include solo la sua posizione rispetto a Dio. Si noti che la giovane carmelitana non evita il paragone con gli altri, fra cui crede di essere l'ultima e la più debole. Da segnalare rimane anche che nel suo intendimento della parola "umiltà", santa Teresa include anche l'umiliazione.

$\mathrm{Ci}$ pare però che le connotazioni più significative siano costituite dai "quattro passi" presentati sopra in cui la semplice accettazione della sua debolezza si trasforma in gioia risultante da una tale condizione, e dalla quale si può in qualche modo trarre profitti. Questi gruppi di connotazioni possono essere considerati come le tappe del percorso fatto per diventare umile. E' da sottolineare che l'intendimento del concetto d'umiltà secondo santa Teresa concilia elementi che si escludono: le 
situazioni, i tratti, i comportamenti difficilmente accettabili (gli errori commessi, la debolezza, la piccolezza, l'impossibilità di fare qualsiasi cosa da soli) acquisiscono nei suoi Scritti un valore positivo.

\section{BIBLIOGRAFIA}

Adamsk a J. 1. (1997), Wszyscy kochać mnie będq. Poznań: Wydawnictwo Totaldruk.

A presjan J.D. (2000), Semantyka leksykalna. Synonimiczne srodki jezyka. Wrocław: Zakład Narodowy im. Ossolińskich.

Baylon C., Fabre P. (1994), La semántica (con ejercicios prácticos y sus soluciones). BarcelonaBuenos Aires-México: Ediciones Paidós.

Dardano M., Trifone P. (1997), La Nuova grammatica della lingua italiana. Bologna: Zanichelli Editore.

Filek O. (red.) (1999), Aby lepiej poznać św. Teresę z Lisieux. Kraków: Wydawnictwo Karmelitów Bosych.

Gaucher G. (1994), Mala święta z Lisieux. Poznań: Wyższe Seminarium Duchowne Karmelitów Bosych.

Gogola J. W. (1997), Mala droga św. Teresy od Dzieciqtka Jezus [in:] Mala droga zupelnie nowa św. Teresy od Dzieciqtka Jezus. Materiały z sympozjum. Kraków: Wydawnictwo Naukowe Papieskiej Akademii Teologicznej.

Gogola J. W. (1999), Sekret św. Teresy z Lisiewx. Elementy duchowości. Kraków: Wydawnictwo Karmelitów Bosych.

Grzegorczykowa R. (1993), Pokora, pycha i pojęcia pokrewne, [in:] J. Bartmiński, M. Mazurkiewicz-Brzozowska (red.), Nazwy wartości, studia leksykalno-semantyczne. Lublin.

Kasperkiewicz K. M. (1983), U podstaw permanentnej formacji. Problematyka chrześcijańskiej pokory. Warszawa: Akademia Teologii Katolickiej, pp. 8-88.

Krzeszowski T. P. (1999), Aksjologiczne aspekty semantyki jzzykowej. Toruń

Léon-Duf our X. (1985), Stownik teologii biblijnej. Poznań-Warszawa: Pallotinum.

Moun in G. (1995), Dictionnaire de la linguistique. Paris: Quadrige, Presses Universitaires de France.

Sainte Thćrc̀se de l'Enfant Jésus (1995), Manuscrits autobiographiques. Office central de Lisieux.

Sainte Thérèse de l'Enfant Jésus (1999), J'entre dans la vie. Derniers Entretiens. Paris: Editions du Cerf.

Sainte Thérc̀se de l’Enfant Jésus (1979), Poésies, un cantique d'amour. Paris: Editions du Cerf.

Sainte Thérìse de l'Enfant Jésus (1983), Une course de géant. Lettres. Paris: Editions du Cerf (lettres dès 9 avril 1888 jusqu'au 25 août 1897).

Teresa di Gesù Bambino (1998), Gli Scritti. Roma: Edizioni OCD.

Tokarski R. (1993), Slownictwo jako interpretacja świata [in:] Encyklopedia kultury polskiej XX wieku, tom II, red. J. Bartmiński. Wroclaw: Wiedza o Kulturze.

Touratier C. (2000), La sémantique. Paris: Armand Colin. 